\title{
In search of the Holy Grail: NF1 mutation analysis and genotype-phenotype correlation
}

As with many great undertakings, the quest sometimes holds more than the find. In this issue, Messiaen et al.' report a mutational "hotspot" in exon $10 \mathrm{~b}$ of the NFI gene. As part of their analysis, 5 of 9 mutations involve a cDNA substitution (Al466G) that predicts a missense mutation (Y489C). However, the substitution of a guanosine at this position creates a splice donor site, which deletes the last 62 bases of exon $10 \mathrm{~b}$ resulting in a stop codon at amino acid-position 489 . The other 4 mutations include a frame-shift $C$ insertion (1465/1466) resulting in a stop codon at position 489 , a 4-bp deletion in intron $10 \mathrm{~b}$ leading to exon dropout of $10 \mathrm{~b}$, which would delete 45 amino acids if a stable protein were translated, and 2 with a T1523C substitution resulting in a L508P missense mutation. Even though the last 2 mutations have not been proven to inactivate the NFI gene product, neurofibromin, their rarity in the unaffected population highly suggests they represent bona fide mutations. All told, the authors screened a total of 232 individuals with NF1, and they identified 9 exon $10 \mathrm{~b}$ mutations. This exon represents approximately $1.6 \%$ of the $N F 1$ coding sequence, therefore there is a 2.4 -fold increased mutation incidence in exon $10 \mathrm{~b}$ over the expected occurrence if mutations occurred randomly throughout the cDNA.

As mutation "hotspots" go, this observation is somewhat lukewarm; however, with respect to NF1, it is as hot as it gets. In a review chapter by Upahyaya and Cooper, two other "hotspots" have been identified. ${ }^{2}$ Exon 31 harbors a CpG dinucleotide that undergoes a recurrent transition (C5839T) resulting in an R1947X codon and premature truncation of translation. ${ }^{3-9}$ As reviewed by Upadhyaya and Cooper, 563 subjects were screened and 10 showed this specific mutation $(2 \%)$, whereas another 10 mutations involving this exon have been identified in an additional 130 subjects, ${ }^{10-12}$ which tallies to 20 mutations in 693 subjects screened or an incidence of approximately $3 \%$. Exon 37 harbors a 4 basepair region between nucleotides 6789 and 6792 that has been shown to be mutated in approximately $1.8 \%$ of subjects screened. . $^{3,3-15}$ Other recurrent mutations are reviewed in Upadhyaya and Cooper, however their numbers do not constitute hotspots.2 Thus, screening the NF1 gene for whole-gene deletions (approximately $5 \%$ of screened individuals with NF1) and exon-specific mutations in exons $10 \mathrm{~b}(4 \%), 31(3 \%)$, and $37(1.8 \%)$ would predict that one could identify approximately $14 \%$ of $N F 1$ germline mutations.

To put this observation into a better perspective, it is important to recognize our limitations in establishing the true denominator in calculations of mutation "hotspots." This would include the number of subjects screened, segment screened, type of mutation analysis, and rigor in demonstrating the vari- ant to be disease-causing. Interestingly, there are a number of "coldspots" or NFl exons for which no mutation has been identified ( $28 \%$ of all exons and $21 \%$ of NFl cDNA). These include exons $1,2,4 \mathrm{a}, 9,14,15,19 \mathrm{~b}, 25,40,43,46,47,48,49$, and the alternative splice exons $9 \mathrm{a}, 23 \mathrm{a}$, and $48 \mathrm{a} .^{-2}$ Thus, the "relative risk" of harboring mutations in exon $10 \mathrm{~b}$ would be less if one subtracts the number of bases representing exons that have never been shown to have a mutation. In such a calculation, the overall expected percentage of mutations in $10 \mathrm{~b}$ becomes approximately $2 \%$ (135 bases in exon $10 \mathrm{~b} / 6792$ bases in the cDNA exons with mutations) rather than $1.6 \%$ ( 135 bases in exon 10b/8601 total cDNA bases). The mutation incidence then goes from a 2.4 -fold increase to a 1.95 -fold increase over what is expected if the mutations were evenly distributed in a random fashion. This analysis suggests that exon $10 b$ harbors more mutations than expected when both RNA and genomic DNA are screened, yet considering the relative lack of $10 \mathrm{~b}$ mutations in the NNFF International NF1 Genetic Analysis Consortium, it still does not provide us with the Holy Grail. Mutational hotspots are useful when identified; however, they need to be carefully defined. As demonstrated by this example, the denominator is an important but often difficult number to determine when one attempts to define the incidence of recurrent mutations.

The outstanding value of the report in this issue by Messiaen et al.' lies in the process by which they screened the NFl gene. Initially the authors screened those subjects for whom lymphoblastoid cell lines were available to perform the Protein Truncation Test (PTT) on total RNA freshly extracted from cell lines. Using random-primed RT-PCR to generate cDNA segments for in vitro transcription and translation (IVTT), they identified 2 of 37 subjects who had an identical sizeshifted peptide in segment 1 (exons 1 through 12b). Upon further analysis they showed that one mutation was a frameshift termination and another was a termination due to aberrant splicing, both involving exon 10b. They then screened 195 subjects at the genomic DNA level for exon $10 \mathrm{~b}$ mutations and identified 7 other mutations, 4 identical to the A $1466 \mathrm{G}$ substitution. As pointed out in the discussion, there is some value in screening both RNA and DNA in determining the molecular etiology of the mutation. In the case of the aberrant splice donor site in exon $10 \mathrm{~b}$, one could have assumed that a missense mutation defined at the genomic DNA sequence level would alter the peptide configuration, whereas, by cDNA analysis it was shown to be a disease-causing mutation due to premature truncation. This is not a novel mutation mechanism, nevertheless it points out the need to fully screen aberrant genomic DNA sequence variants for functional alterations at both the 
mRNA and protein levels. Unfortunately, analysis of the peptide function is not available in the clinical setting, but mRNA can, and should be evaluated as part of screening protocols. Indeed, the NNFF International NFl Genetic Analysis Consortium includes a number of mRNA splicing mutations, 17 reported in 11 published studies, ${ }^{2}$ and, given that $N F 1$ mRNA has not been screened routinely, it is likely that a substantial percentage of NFl mutations result from aberrant splicing due to mutations in exons in addition to introns, as shown in this report.'

Although essential in comprehensive mutation analysis, NF1 CDNA screening still falls short in one important arena, and it is exemplified in the Messiaen et al. ${ }^{1}$ report. The authors have not yet had the opportunity to determine the status of splicing of the Al466G mutation in tissue that represents the NFl phenotype. It will be imperative to screen tissue from the subiects who harbor this mutation, as it becomes available, to demonstrate that the aberrant splicing of NFl primary transcript in lymphocytes, which is a tissue not involved in the NFI phenotype, is also aberrantly spliced in NFl-affected tissue. Such a recommendation is not restricted to this report or to $\mathrm{NF} 1$ in general, but applies to most genes that are screened for mutations using lymphocyte or some other ectopic RNA expressed from tissue that is not usually involved in the phenotype of the disorder.

Regardless of the nature of NF1 mutations and their incidence in a gene that is commonly mutated in the human population, one must still ask, of what value is this information? Clearly, it is an important step in assessing genotype-phenotype correlations and in providing insight toward the pathophysiology of NF1. But, in this age of managed health care and shrinking research dollars, is NF1 mutation detection a worthwhile endeavor? I posit that knowledge of the NFI mutation plays a minimal, if any, role in clinical management of affected families. Hotspots or not, mutation detection does not provide insight that physical examination and experienced genetic counseling does not already provide. NFl remains a clinical diagnosis based on tried and true criteria ${ }^{16}$ that have been confirmed as valid and useful. ${ }^{17}$ The few instances in which knowledge of the constitutional mutation modifies clinical management does not warrant the use of clinical and research dollars to satisfy a "need to know." In some instances, presymptomatic testing in families in which the mutation is known could alter the early childhood surveillance of childhood complications, especially optic nerve gliomas. However, most medical centers are no longer performing routine MRI screening unless there are ophthalmological or neurological symptoms. The tradeoff of clinically following at-risk individuals through age 10 years, which includes dealing with the anxiety raised by the possibility of having a diagnosis with age-dependent onset of signs, should be weighed carefully against the value of NF1 mutation detection screening as a diagnostic test. Presently, a concerted effort toward a hypothesis-driven, protocol-based approach to address specific questions regarding the pathophysiology of NFl is preferable to broad-based, unfocused NFl mutation screening, even if performed in the research arena.
In closing, Messiaen et al. ${ }^{1}$ have clearly demonstrated the importance of screening NFI mutations at both the RNA and DNA level, and they have identified a recurrent mutation that may be important in streamlining mutation analysis. They, like others, could not detect a genotype-phenotype correlation with respect to $N F 1$ mutations involving exon 10b. Their work points out the importance of looking elsewhere, beyond the NF1 mutant allele and with other techniques, to explain the variability of clinical expression of this complex and intriguing condition. ${ }^{18}$ In addition, it shows the difficulty in presenting complete phenotypes of subjects enrolled from multiple centers as part of a comprehensive report to be published in journals that are competitive for space. Nevertheless, to be most effective, a presentation of phenotypes, including both positive and negative findings, with respect to age would be important in compiling the compendium needed to exhaustively examine, and maybe put to rest, the notion that any exon-specific NF1 mutation plays a significant role in genotype-phenotype correlation. Hopefully, a format that encourages the collection and reporting of robust phenotype analysis of genetic conditions can be developed in this age of electronic publishing to assist in engaging the collective readership in searching for the Holy Grail, in this instance genotype-phenotype correlation.

David Viskochil, MD, PhD Associate Professor in Pediatrics Chief, Division of Medical Genetics University of Utah Salt Lake City, Utah

\section{References}

1. Messiden LM, Callens T, Roux KJ, Mortier GR, De Paepe A, Abramowicz M, Pericak-Vance MA, Vance JM, Wallace MR. Exon 10b of the NFI gene represents a mutational hotspot and harbors a recurrent missense mutation $\mathrm{Y} 489 \mathrm{C}$ associated with aberrant splicing. Genetics in Medicine 1999:1:248-253.

2. Upadhyaya $\mathrm{M}$, Cooper DN. The mutational spectrum in neurofibromatosis 1 and its underlying mechanisms. In: Upadhyaya M, Cooper DN, editors. Neurofibromatosis type 1: From genotype to phenotype. Oxford: BIOS Scientific Publishers Ltd., 1998:65-88.

3. Abernathy CR, Rasmussen SA, Stalker HJ, Zori R, Driscoll DJ, Williams CA, Kousseff BG, Wallace MR. NF1 mutation analysis using a combined heteroduplex/ SSCP approach. Hum Mutat 1997;9:548-554.

4. Ainsworth PI, Rodenhiser DI, Costa MT. Identification and characterization of sporadic and inherited mutations in exon 31 of the neurofibromatosis (NFI) gene. Hum Genet 1993;91:151-156.

5. Cawthon RM, Weiss R, Xu G, Viskochil D, Culver M, Stevens I, Robertson M, Dunn D. Gesteland R, O'Connell P, White R. A major segment of the neurofibromatosis type 1 gene: $:$ DNA sequence, genomic structure, and point mutations. Cell 1990;62: 193-201.

6. Estivill X, Lazaro C, Casals T, Ravella A. Recurrence of a nonsense mutation in the NFl gene causing neurofibromatosis type 1. Hum Genet 1991;88:185-188.

7. Horiuchi T, Hatta N, Matsumoto M, Ohtsuka H, Collins FS, Kobayashi Y, Fujita S Nonsense mutations at Arg-1947 in two cases of familial neurofibromatosis type 1 in Japanese. Hum Genet 1994;93:81-83.

8. Hudson I. Wu CL, Tassabehji M, Summers EM, Simon S, Super M, Donnai D, Thakker N. Novel and recurrent mutations in the neurofibromatosis type 1 (NFI) gene. Hum Mutat 1997;9:366-367.

9. Valero MC, Velasco E, Moreno F, Hernandez-Chico C. Characterization of four mutations in the neurofibromatosis type 1 gene by denaturing gradient gel electrophoresis (DGGE). Hum Molec Genet 1994;3:639-641.

10. Ainsworth P, Rodenhiser D, Stuart A, Jung J Characterization of an intron 31 splice junction mutation in the neurofibromatosis type 1 (NFI) gene. Hum Molec Genet 1994:3:1179-1181. 
11. Hatta N, Horiuchi T, Watanabe I, Kobayashi Y, Shirakata, Y, Ohtsuka H, Minami T, Ueda K, Kokoroishi T, Fujita S. NFI gene mutations in Japanese with neurofibromatosis 1. Biochem Biophys Res Commun 1995;21 2:697-704.

12. Zhong I, Spiegel R, Boltshauser E, Schmid W. Two novel mutations: $5108 \mathrm{del} A G$ and 5816insG in the NFI gene detected by SSCP analysis. Hum Molec Genet 1993;2: 1491-1497.

13. Boddrich A, Robinson PN, Schulke M, Buske A, Tinschert S, Nurnberg P. New evidence for a mutation hotspot in exon 37 of the NFI gene. Hum Mutat 1997;9: $374-377$.

14. Messiaen L, Callens T, DePaepe A, Craen M, Mortier G. Characterization of two different nonsense mutations, C6792A and C6792G, causing skipping of exon 37 in the NFl gene. Hum Genet 1997;101:75-80

15. Upadhyaya M, Maynard J, Osborn M, Harper PS. Six novel mutations in the neurofibromatosis type 1 (NF1) gene. Hum Mutat 1997;10:248-250.

16. NIH Consensus Development Conference Neurofibromatosis. Arch Neurol 1988; 45:575-578.

17. Gutmann DH, Aylsworth A, Carey J, Korf B, Marks J, Pyeritz RE, Rubenstein A, Viskochil D. The diagnostic evaluation and multidisciplinary management of neurofibromatosis 1 and neurofibromatosis 2. JAMA 1997;278:51-57.

18. Carey JC and Viskochil DH. Neurofibromatosis type 1: A model condition for the study of the molecular basis of variable expressivity in human disorders. Am J Med Genet (Semin Med Genet) 1999;89:7-13.

\section{Erratum}

The article by Yang et al, published in the July/August issue of Genetics in Medicine, omitted a coauthor. The correct authors and their affiliations are as follows:

Huiying Yang, $\mathrm{MD}, \mathrm{PhD}^{1}$, Jeffery D. Ohmen, $\mathrm{PhD}^{2}$, Yuanhong Ma, $\mathrm{PhD}^{2}$, L. Gordon Bentley ${ }^{2}$, Stephan R. Targan, $\mathrm{MD}^{3}, \mathrm{Nathan}$ Fischel-Ghodsian, $\mathrm{MD}^{1}$, and Jerome I. Rotter, $\mathrm{MD}^{1}$

From the ${ }^{1}$ Division of Medical Genetics, Departments of Medicine and Pediatrics, Steven Spielberg Pediatric Research Center, CedarsSinai Research Institute, Cedars-Sinai Medical Center, Los Angeles, California; the ${ }^{2}$ Parke-Davis Laboratory for Molecular Genetics (PDLMG), Alameda, California; and the ${ }^{3}$ Inflammatory Bowel Disease Center, and Division of Gastroenterology, Department of Medicine, Cedars-Sinai Research Institute, Cedars-Sinai Medical Center, Los Angeles, California. 